\section{TISSUE-RESIDENT CELLS}

\section{$T_{R M}$ cells hit the road}

Sci. Immunol. https://doi.org/10.1126/sciimmunol. aav8995 (2019)

Tissue-resident memory $\mathrm{T}$ cells $\left(\mathrm{T}_{\mathrm{RM}}\right.$ cells) serve important roles in maintaining immunological defence at tissue barriers and by and large keep to their given tissue environment. In Science Immunology, Gratz and colleagues use a combination of human skin explants and xenotransplantation into immunocompromised mice to demonstrate that a small subset of $\mathrm{CD} 4^{+} \mathrm{T}_{\mathrm{RM}}$ cells can egress the skin and re-enter the circulation. These blood-borne cells have many of the phenotypic and transcriptomic characteristics of classic $\mathrm{T}_{\mathrm{RM}}$ cells but downregulate the C-type lectin CD69 to facilitate egress from the skin. $\mathrm{T}_{\mathrm{RM}}$ cells that have exited the skin can migrate systemically to seed distal skin sites. The precise trigger for this $\mathrm{T}_{\mathrm{RM}}$ cell migration and its physiological consequences remain unclear but might have important implications for the dissemination of immunity or immunopathology.

https://doi.org/10.1038/s41590-019-0487-x

\section{CD24 IN TUMORS}

\section{Inhibiting phagocytosis}

Nature https://doi.org/10.1038/s41586-019-1456-0 (2019)

CD24 is an inhibitory molecule with high expression on multiple tumor cell types, including ovarian and triple-negative breast cancers. In Nature, Barkal et al. show that the inhibitory receptor Siglec-10 expressed on tumor-associated macrophages suppresses their ability to phagocytose CD24 ${ }^{+}$tumor cells. The cytokines IL-10 and TGF- $\beta$ induce Siglec-10 expression by tumor-associated macrophages. Disrupting the CD24-Siglec-10 interaction either by genetic loss of CD24 or SIGLEC10 or by monoclonal antibody-mediated blockade increases macrophage-dependent phagocytosis of tumor cells, which indicates that CD24-Siglec-10 binding provides a 'don't eat me' signal. Combined blockade of CD24 and CD47 (another 'don't eat me' molecule) synergistically enhances the ability of macrophages to phagocytize primary human tumor cells in vitro. Mouse macrophages also show enhanced phagocytosis of CD24-deficient tumor cells in vivo. These findings suggest that targeting CD24-Siglec-10 interactions might prove beneficial for immunotherapy in cancer.

https://doi.org/10.1038/s41590-019-0485-Z

\section{INNATE MEMORY}

\section{DCs remember}

Nat. Commun. https://doi.org/10.1038/s41467019-10486-5 (2019)

Immunological memory has now been well characterized in innate immune

Macrophages contribute to wound healing. In Immunity, Gallagher and colleagues show that the methyltransferase Setdb2, which induces repressive H3K7me3 chromatin modifications, controls the transition of inflammatory macrophages to a reparative phenotype during wound healing. Expression of Setdb2 is increased in wound macrophages at the stage of wound healing (day 5). Setdb2-deficient mice have delayed wound healing and higher expression of the genes encoding the cytokines IL-1 $\beta$ and TNF, with less H3K9me3 in the promoters of these genes. There is also less $\mathrm{H} 3 \mathrm{~K} 9 \mathrm{me} 3$ at the promoter of the gene encoding xanthine dehydrogenase, which converts xanthine to uric acid. The cytokine IFN- $\beta$ increases the expression of Setdb2 in wound macrophages. Mice with diet-induced obesity have lower IFN- $\beta$ expression in the wound, with less Setdb2, less $\mathrm{H} 3 \mathrm{~K} 9 \mathrm{me} 3$ at NF-KB-responsive promoters, increased production of pro-inflammatory cytokines and more uric acid (an activator of the NLRP3 inflammasome) in wound macrophages. Treatment with an inhibitor of uric acid production improves wound healing in obese mice.

cells such as natural killer cells and macrophages; however, less is known about dendritic cells (DCs) in this context. In Nature Communications, Wormley and colleagues use a model of protective intranasal vaccination and fungal challenge to investigate the potential of DC memory responses. DCs isolated from the spleen weeks after clearance of the fungus via the protective vaccine show enhanced production of pro-inflammatory cytokines after re-challenge with fungus. Enhanced DC 'memory' responses are dependent on histone modifications. Interestingly, the DC memory response has some elements of pathogen specificity, although the mechanism involved remains unclear. These findings may have implications for the tailored design of vaccines.

https://oi.org/10.1038/s41590-019-0486-y

\section{NEUROIMMUNOLOGY}

\section{Anticipatory immunity}

Cell https://doi.org/10.1016/j.cell.2019.06.022 (2019)

TRVP1 $1^{+}$peripheral sensory neurons are specialized to detect pain and respond to pathogen-associated molecules such a Candida albican beta glucan and bacterial toxins and are required for the initiation of type 17 inflammation. In Cell, Kaplan and colleagues show that optogenetic stimulation of the TRPV $1^{+}$ neurons is sufficient to trigger type 17 inflammation in the skin in the absence of additional damage- or pathogenassociated stimuli. The inflammatory response is characterized by the infiltration of $\gamma \delta \mathrm{T}$ cells, $\mathrm{CD} 4^{+} \mathrm{T}$ cells and neutrophils and secretion of the cytokines IL-23, IL-6 and TNF and is dependent on release of the neuropeptide CGRP $\alpha$ from the TRPV $1^{+}$ neurons and on IL-17A. Activation of TRPV $1^{+}$neurons via optogenetic stimuli or infection with C. albicans induces a nerve reflex that triggers the production of type 17 cytokines and provides increased immunity to C. albicans at adjacent sites, indicative of anticipatory immunity to infection.

https://doi.org/10.1038/s41590-019-0483-1

Laurie A. Dempsey, Zoltan Fehervari and loana Visan 